Acta Theriologica 38 (3): 305 - 314, 1993.

PL ISSN $0001-7051$

\title{
Estimating population density of moles Scalopus aquaticus using assessment lines
}

\author{
Gregory D. HARTMAN* and John D. KRENZ
}

\begin{abstract}
Hartman G. D. and Krenz J. D. 1993. Estimating population density of moles Scalopus
\end{abstract} aquaticus using assessment lines. Acta theriol. 38: $305-314$.

Density was estimated for three populations of eastern moles Scalopus aquaticus Linnaeus, 1758, in South Carolina using a trapping grid and assessment lines. Assessment line data were based upon the repair of holes punched into surface tunnels of the animals. Using a new method of analysis for assessment line data, a logistic curve was fitted to the data. Density estimates based upon these analyses were (95\% confidence intervals in parentheses) $3.02(1.90-4.98), 2.73(1.48-12.52)$, and 1.71 $(0.86-2.69) \mathrm{moles} / \mathrm{ha}$. In previous studies using assessment line data to estimate density, obtaining confidence intervals for the density estimate presented theoretical problems. In this study, Monte Carlo procedures were used to obtain an estimate of the approximate $95 \%$ confidence intervals for density. The analytical methods used in the present study eliminate the concepts of boundary strip width (strict sense) and the occurrence of distinctive zones with constant capture probabilities, and as such, represent important conceptual improvements of the assessment line density estimation method.

Department of Biology \& Museum of Southwestern Biology, University of New Mexico, Albuquerque, NM 87131, USA (GDH); Savannah River Ecology Laboratory, Drawer E, Aiken, SC 29802, USA (JDK; address for reprint requests)

Key words: Scalopus aquaticus, Talpidae, assessment lines, population density, South Carolina

\section{Introduction}

Various methods have been proposed to estimate the densities of animal populations (see Smith et al. 1975, White et al. 1982, Anderson et al. 1983, Wilson and Anderson 1985, for reviews). Assessment lines (Wheeler and Calhoun 1968, Smith et al. 1971) have been used to estimate population densities of numerous species of small mammals (see Smith et al. 1971, Swift and Steinhorst 1976). With this method, animals trapped in an area or along a census line either are removed or effectively are removed by marking. After trapping, the presence of remaining or unmarked animals is monitored along transects (assessment lines) that intersect the trapping area. These data then are used to estimate the proportion of animals removed during trapping and the size of the area that effectively was trapped.

\footnotetext{
"Present address: Environmental Sciences Section, Savannah River Technology Center, Bldg. 773-42A, P.O. Box 616, Aiken, SC 29802, USA
} 
An advantage to using assessment lines is that a smaller trapping grid is required than is necessary for density estimation techniques such as nested sub-grids (Otis et al. 1978) or the trapping web (Anderson et al. 1983); however, the theoretical and statistical bases of the method have been considered to be problematic (Swift and Steinhorst 1976, Wilson and Anderson 1985). Many of the past problems associated with the analysis of assessment line data can be linked to the idea that trapping will result in discrete and recognizable zones. The probability of capturing an animal was envisioned as a stepped function of the distance from the edge of the trap area. This conceptual framework makes the estimations of the effective area trapped and density tractable, but probably is too unrealistic. A model of animal removal representing a continuum of capture probabilities is more appropriate than the use of a discrete zone-type model, because the probability of capturing an animal whose home range partially overlaps the trapping area should vary as some function of the degree of overlap (Swift and Steinhorst 1976).

One limitation of the treatment of assessment line data in most previous studies was the regression of the accumulative numbers of captures on distance. Accumulative data are not independent, and therefore, any confidence intervals calculated for the boundary strip width estimate are biased (Smith et al. 1971, 1975). In addition, because there is no consensus as how to fit three regression lines (corresponding to complete, partial, and no removal) to assessment line data (O'Farrell et al. 1977), there has been no objective method for determining the position of the edge of the area of effect due to trapping (Swift and Steinhorst 1976).

Moles (Insectivora, Talpidae) primarily are predators of invertebrates and occur at densities much lower than those of most rodents of comparable mass. As such, estimating the population density of moles via some of the more commonly used and popular methods (e.g. sub-nested grids or trapping webs) require that very large areas be surveyed in order to obtain reliable estimates. Because of their low population densities, subterranean habits, and difficulty of capture, moles represent an extreme in terms of the cost and effort required to capture relatively large numbers of animals in a systematic manner. There are live traps for moles that work very well (Moore 1940, Yates and Schmidly 1977, Gorman and Stone 1990), however, these are expensive to manufacture in large numbers and require considerably more time to set than do kill traps. These factors can make the reliable estimation of mole population densities prohibitively expensive and/or logistically impractical for most investigators.

In this paper, we report density estimates for eastern moles Scalopus aquaticus Linnaeus, 1758, obtained from assessment line data that are based upon an index of animal activity. An alternative analytical model is presented wherein the removal of animals is considered as a continuous (logistic) function rather than as occurring in discrete steps or zones. We also employ a bootstrap procedure that provides confidence intervals for the density estimate. 


\section{Materials and methods}

The study was conducted on the United States Department of Energy's Savannah River Site located near Aiken, South Carolina. Habitats consisted of pine plantations with intermittently occurring hardwoods. Soils were sands or sandy loams covered with a layer of pine needles and other debris. There were three study localities, hereafter referred to as grids A, B, and C. At each of the three grids, there was a 7-day trapping period, immediately followed by a 6 -day post-trapping assessment period.

Trapping was conducted at grids A and B during spring, and corresponded to the annual breeding season of S. aquaticus in the region. Trapping at grid C was conducted during July, approximately 1.5 months after young of the year began to appear in tunnel systems. Moles were captured using Victor brand harpoon-type kill traps. Two traps were set at each station of a 14 by 14 square grid (10.6 ha) with an interstation distance of $25 \mathrm{~m}$. Traps were not moved from their locations during the 7-day trapping period.

Assessment lines had stations at $10-\mathrm{m}$ intervals and extended $200 \mathrm{~m}$ out from the edge of the grid and $150 \mathrm{~m}$ into the grid (Fig. 1). Ten holes were punched in surface tunnels at each assessment line station. Holes were at approximately $1-\mathrm{m}$ intervals along a line perpendicular to the assessment line, with five holes on each side. Holes were individually marked. Eastern moles are particularly intolerant of any breaks in their tunnels and these are repaired soon after they are encountered (G. D. Hartman, pers. observ.). After six days, the number of repaired holes at each assessment line station was recorded. Six days were allowed to pass before collecting the data because $S$. aquaticus do not traverse their entire home ranges during any single period of activity and shift centers of activity within their home ranges over a period of several days (Harvey 1976).

For each grid, data from all assessment lines were pooled by 10 - $\mathrm{m}$ distance classes. The areas represented by the distance classes consisted of a series of concentric square bands, each $10 \mathrm{~m}$ wide with the side assessment line stations at the centers of the bands. Corner assessment lines had the same length and number of stations as did the assessment lines that passed perpendicular through the sides of the grids (Fig. 1). Stations on corner assessment lines were included in the distance class band within which they occurred. The effect of trapping was absent or negligible at distances greater than $100 \mathrm{~m}$ from the grid, and data from stations at these distances were used to calculate a mean frequency of repair of holes for each study area. Using this method, population size and density thus

Fig. 1. Trapping grid and assessment line layout used in the study. Open circles represent stations of the trapping grid. There were three corner assessment lines per grid because unsuitable habitat lay within $100 \mathrm{~m}$ of one corner of each grid.

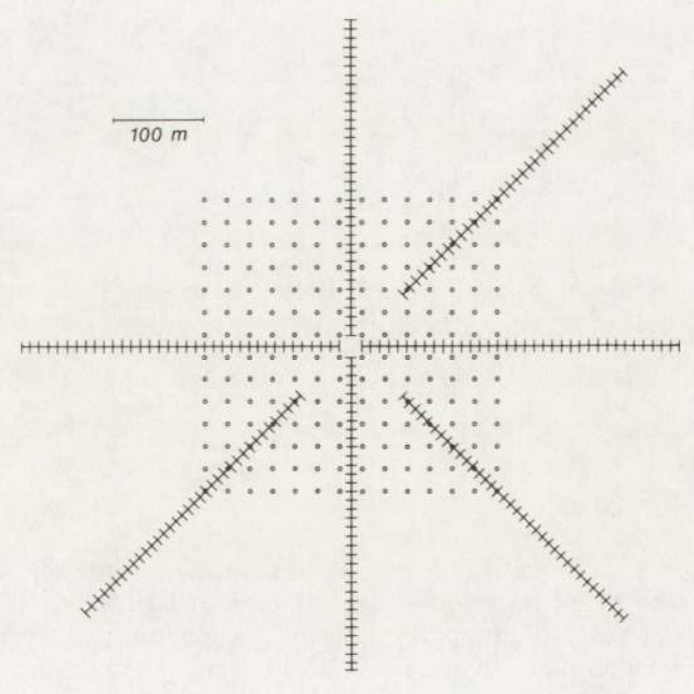


were estimated for the same area for each grid, namely 27.6 ha; we refer to this as the estimation area $\left(A_{\mathrm{e}}\right)$.

The data were subjected to non-linear regression analysis using a logistic model, with distance as the independent variable and the number of repaired holes as the dependent variable. Regressions were performed using the nonlinear least-squares regression (NLIN) procedure of SAS (SAS Institute 1985). To compensate for heteroscedasticity in the data, an iteratively reweighted regression was used. The constraints imposed upon the logistic model were as follows: (1) the upper asymptote was no greater than the mean frequency of hole repair, and (2) the lower asymptote was not less than zero.

A three-dimensional model was used to estimate density as follows. The mean frequency of hole repair $\left(\bar{Y}_{\mathrm{r}}\right)$ was viewed as the height of a plane overlying the estimation area $\left(A_{\mathrm{e}}\right)$. The volume of the solid underlying this plane $\left(V_{\mathrm{t}}\right)$ was equal to $Y_{\mathrm{r}}$ multiplied by $A_{\mathrm{e}}$, had units equal to percent repaired holes . ha, and was proportional to the number of animals present in $A_{\mathrm{e}}$. The per animal rate of hole repair was assumed to be independent of changes in density that occurred due to trapping.

The removal of animals decreased the frequency of hole repair and resulted in a portion of $V_{\mathrm{t}}$ being "scooped" out (Fig. 2). The volume that remained $\left(V_{\text {nc }}\right)$ was assumed to be proportional to the number of animals present in $A_{\mathrm{e}}$ that were not captured during trapping, and was determined using the position of the regression curve. $V_{\mathrm{nc}}$ was calculated as:

$$
V_{\mathrm{nc}}=\sum_{i=1}^{n} 4\left(W_{i}\right)\left(L_{i}\right)\left(Y_{i}\right)
$$

where $W_{i}$ is the width of distance class band $i$ ( $10 \mathrm{~m}$ for each band in the present study), $L_{i}$ is the length of the midline of the distance class band, $Y_{i}$ is the value of the regression curve corresponding to the midpoint of the distance class band, and $n$ is the number of distance class bands.

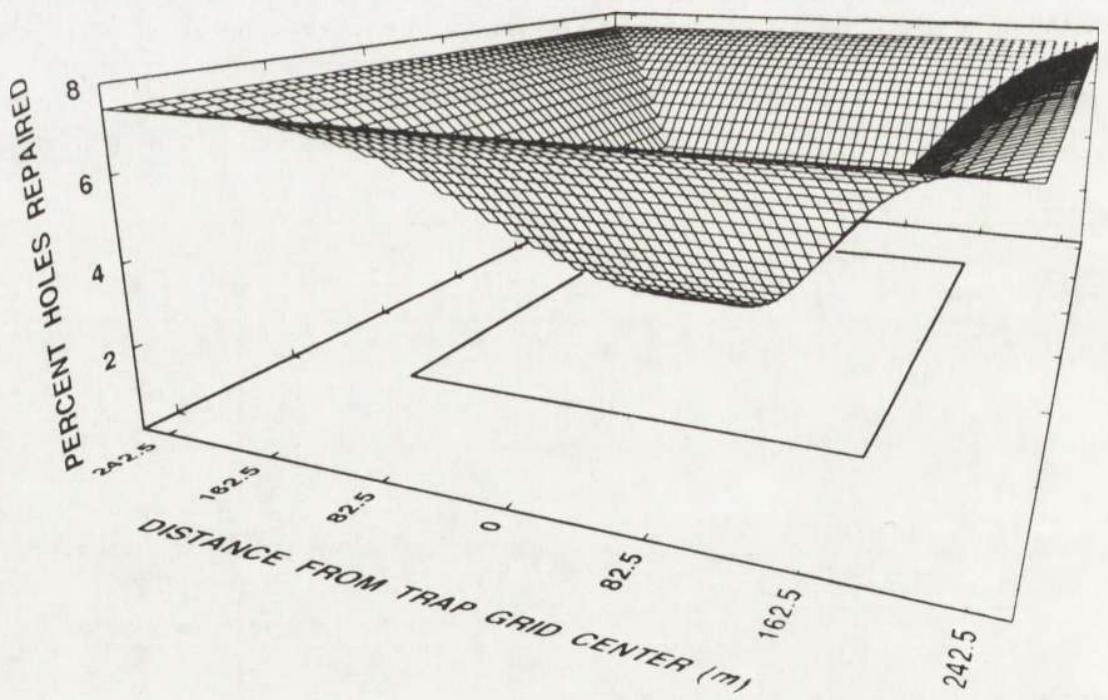

Fig. 2. Percent holes repaired over the estimation area of grid A. This surface was constructed from the fitted regression line depicted in Fig. $3 . V_{n c}$ is the volume beneath the surface, and is used to calculate $\hat{P}_{\mathrm{r}}$, the proportion of the population removed. The $x$ and $y$ axes are identical. The square located beneath the surface represents the perimeter of the trap grid. 
The proportion of the population removed from the estimation area by trapping $\left(\hat{P}_{\mathrm{r}}\right)$ was estimated as:

$$
\hat{P}_{\mathrm{r}}=V_{\mathrm{t}}-V_{\mathrm{nc}} / V_{\mathrm{t}}
$$

The estimate of the number of animals initially present within the estimation area was:

$$
\hat{N}_{\mathrm{a}}=N_{\mathrm{c}} / \hat{P}_{\mathrm{r}}
$$

where $N_{\mathrm{c}}$ is the number of animals captured on the grid. Density was estimated as:

$$
\hat{D}=\hat{N}_{\mathrm{a}} / A_{\mathrm{e}} \text {. }
$$

Ninety-five percent confidence intervals (CI) for the density estimates were obtained using Monte Carlo procedures. In each iteration, the original 70 data points (each having a value of either 0 or 1 ) for each distance class were randomly sampled with replacement 70 times and the resulting data set was used to estimate population density as described above. This procedure was repeated 200 times, providing a distribution of 200 density estimates for each grid, which then enabled 'bootstrap' estimation of confidence intervals for the original density estimates.

\section{Results}

The assessment line data and fitted regression curve for grid $\mathrm{A}$ are shown in Fig. 3. Coefficients of determination $\left(r^{2}\right)$ for the regressions of the original data for grids $\mathrm{A}, \mathrm{B}$, and $\mathrm{C}$ were $0.58,0.16$, and 0.54 , respectively. All regressions were significant at the $p \leq 0.005$ level $(F$-test, $\mathrm{df}=4,22)$. Density estimates based upon these regressions were 1.71, 2.73 and 3.02 moles/ha (Table 1). For two of the grids (A and $\mathrm{C}$ ), the mean population size and density estimates obtained from bootstrap analyses agreed well with those obtained from the original analyses. Population parameter estimates for grid B obtained from the original regressions and bootstrap analyses did not agree well; however, the estimates of both the proportion of

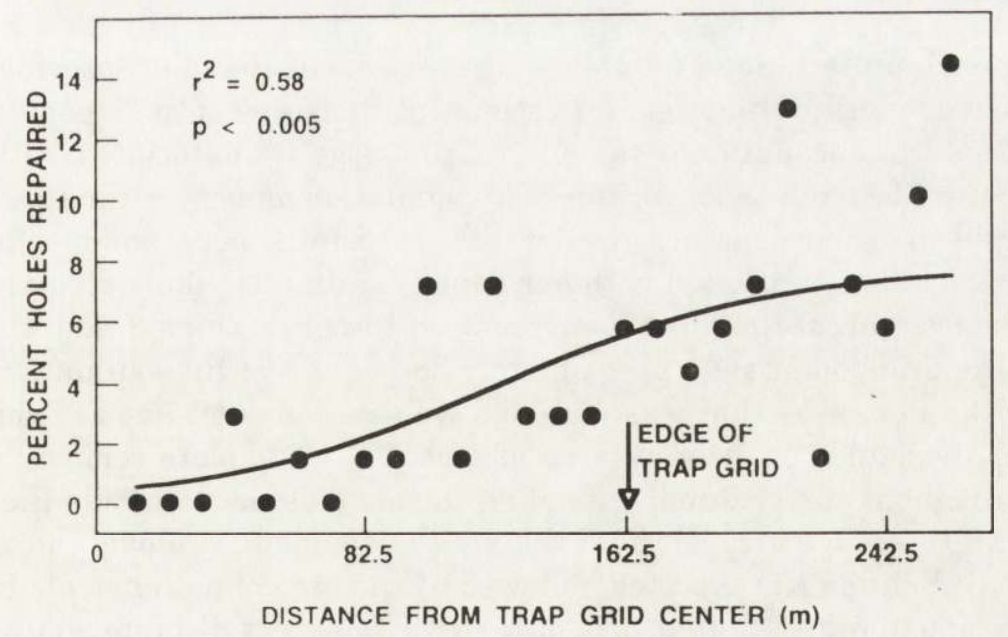

Fig. 3. Percent of holes repaired along assessment lines vs distance from the center of the trapping area for grid A. The logistic curve was fitted to the data using iteratively reweighted non-linear least-squares regression. Each data point represents a mean $(n=70)$ for a single distance class band. 
Table 1. Estimates of mole population parameters and descriptive statistics obtained by non-lïnear regression of assessment line data using a logistic model. $N_{\mathrm{c}}$ represents the actual number of moles captured at a grid. Estimates are presented for the proportion of the population removed by trapping $(\hat{P})$, population size $(\hat{N})$, density $(\hat{D})$ (moles/ha), and approximate $95 \%$ confidence intervals (CI) for density. Values in columns with the subscript 'a' were obtained using the actual data; those with a subscript of 'b' represent values obtained from bootstrap analyses. Confidence intervals were considered as the 2.5th and 97.5th percentiles of the distribution obtained from bootstrap analysis for each grid.

\begin{tabular}{lccccccr}
\hline Grid & $N_{\mathrm{c}}$ & $\hat{P}_{\mathrm{a}}$ & $\hat{P}_{\mathrm{b}}$ & $\hat{N}_{\mathrm{a}}$ & $\hat{D}_{\mathrm{a}}$ & \multicolumn{1}{c}{$\hat{D}_{\mathrm{b}}$} & $\mathrm{CI}\left(\hat{D}_{\mathrm{b}}\right)$ \\
\hline A & 22 & 0.26 & 0.28 & 83.19 & 3.02 & 2.96 & $1.90-4.98$ \\
$\mathrm{~B}$ & 20 & 0.27 & 0.21 & 75.36 & 2.73 & 4.84 & $1.48-12.52$ \\
$\mathrm{C}$ & 12 & 0.25 & 0.32 & 47.26 & 1.71 & 1.52 & $0.86-2.69$ \\
\hline
\end{tabular}

the population removed and density based upon the original data were very similar for all three grids (Table 1).

For each grid, the distribution of density estimates obtained when the data were bootstrapped were significantly non-normal $(p<0.05,0.01$, and 0.01 for $\mathrm{A}$, $\mathrm{B}$, and $\mathrm{C}$, respectively). Therefore, the approximate $95 \%$ confidence intervals (CI) for the density estimates were considered as the 2.5th and 97.5th percentiles of the distributions (Table 1), rather than \pm 2 standard deviations of the mean (Caswell 1989). Estimated 95\% CI for the density estimates were much smaller for grids A and C than for grid B (Table 1).

\section{Discussion}

The hole punching technique for obtaining assessment line data provides both a low-cost and time-efficient method for estimating densities of mole populations. A problem that was encountered was the rather large CI associated with each density estimate. Although large variances in population density estimates generally are a problem when densities are low (M. H. Smith, pers. comm.), a large portion of the variation associated with our density estimates likely resulted from not having removed all of the animals present on the grids (Figs 3 and 4).

The trapping grid comprised approximately 38 percent of the estimation area, yet the highest value of $Y_{\mathrm{r}}$ that was obtained was only about $27 \%$. Efficient and intense trapping should result in an area of essentially complete removal of animals both throughout the grid and extending to some distance outside the edges of the grid (Smith et al. 1971). Beyond the area of complete removal, an area of partial removal of animals is expected, followed by the area of no removal. However, it is important to reiterate that it is unrealistic to expect discrete zones corresponding to complete, partial, and no removal of animals extending equidistant around the edges of a grid. 
Fig. 4. Numbers of moles captured during seven days of trapping at each of three grids. Trapping periods 1 through 3 each represent two days of trapping, and period 4 represents one day of trapping. Only the capture data for grid A suggest that most of the resident moles had been captured; however, assessment line data indicated that moles still were present within this grid (refer to Fig. 3).

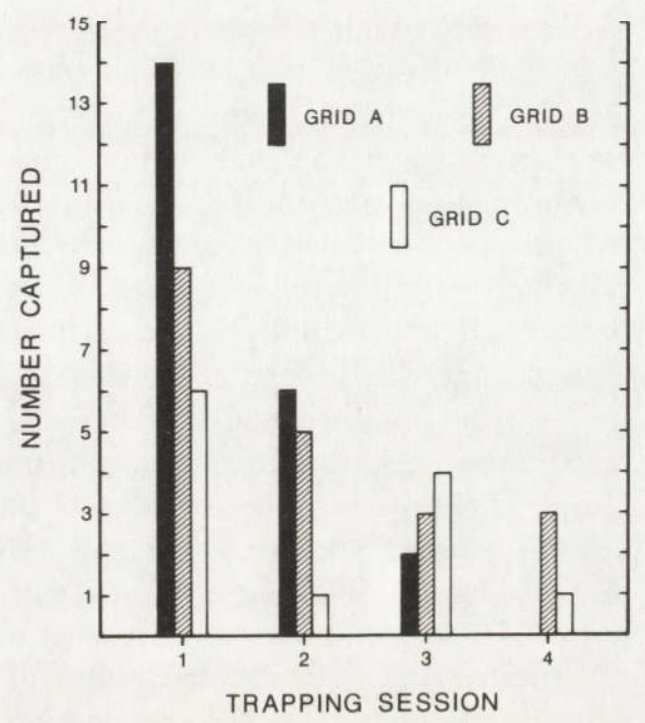

Because the proportion of moles removed by trapping was relatively low for all three grids in our study, the logistic curve was fitted to a larger number of distance classes than if there had been complete or nearly complete removal of animals from the grid area. This resulted in greater confidence interval widths because the assessment line technique for estimation of population density is sensitive to the distance over which the trapping effect diminishes. Had there been a more complete removal of animals within each grid, the area corresponding to partial removal of animals would have been much smaller, with the result that the slope at the inflection point of the logistic curve would have been much steeper than those that were observed. Although traps were placed selectively (based on the overall appearance of tunnels and the feel of tunnel walls), they were not placed on tunnels that previously had been determined to be in use by moles. Greater efficiency of removal trapping could be accomplished by punching holes in a number of tunnels at each trapping station a few days before trapping begins to assess if a tunnel is regularly being used by a mole, a method analogous to pre-baiting of traps.

In the area of no measurable effect due to trapping, the rate of hole repair was highly variable among distance class bands (Fig. 3). This variation likely was the result of (1) sampling error as a result of the density and dispersion of assessment line stations across the estimation area, and (2) the density and dispersion of moles. The total linear distance covered by holes punched at assessment line stations remained essentially constant from one distance class band to another, i.e. about $70 \mathrm{~m}$. Although proportionately less of each successively larger distance class band was 'sampled' by the assessment line stations, the difference in the percent coverage of the largest and smallest distance class bands used to estimate 


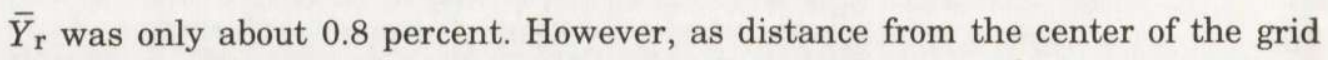
increased, the distance between corresponding stations on adjacent assessment lines increased; this resulted in large portions of the estimation area not having been sampled by the assessment lines (Fig. 1).

Some moles present in the estimation area likely influenced the data more than did others. Harvey (1976) reported that female S. aquaticus in Kentucky had relatively circular home ranges, whereas the home ranges of males were more linear and approximately three times the size of those of females. The annual home range sizes were estimated to be about 1.0 ha for males and 0.3 ha for females. No data are available on dispersion patterns or home range/territory sizes in S. aquaticus in South Carolina, however, an area of activity for any male or female mole during the assessment period likely would have been much less the annual home range size. Given the layout of assessment line stations and the sizes and shapes of mole home ranges, some mole home ranges likely had no overlap with a station whereas other moles may have visited two or more stations. For future studies, a greater frequency of assessment line stations in each distance class band and a more even dispersion of stations throughout the estimation area should result in a reduction of the among-band variation in the estimated frequency of hole repair.

The application of the logistic model to areas of partial removal of animals represents a conceptual extension and improvement of the continuum model used by Swift and Steinhorst (1976). In their study, a linear regression of the proportion of the population removed vs area was used to calculate $\hat{P}_{\mathrm{a}}$ and boundary strip width. It is not clear whether Swift and Steinhorst (1976) had areas of complete removal in their study, or whether data corresponding to areas of complete removal were used in their linear regression. Nevertheless, Swift and Steinhorst's model employed the estimation of a discrete boundary strip width because animal removal decreased as a quadratic function of distance going out from the grid, and at the distance corresponding to the edge of the boundary strip, the proportion of the population removed instantaneously became zero. Fitting the logistic curve to the data is a more realistic way to model animal removal for two reasons. First, using the logistic model assumes that the probability of capturing an animal with a circular home range is directly proportional to the amount of overlap between the home range and the grid. Secondly, the proportion of the population removed approaches zero asymptotically rather than instantaneously becoming zero as distance from the grid increases.

There are some problems associated with a square trapping grid when assessment lines are used to estimate density, and for future studies, a circular trapping area seems preferable. Because of different trap densities, the effect of trapping likely extends farther out (but to an unknown extent) from the side of a square grid than from a corner. A circular trapping area provides a uniform density of traps along the perimeter, and more importantly, the perimeter is equidistant from the center. With a square grid, corner assessment lines cross the distance class 
bands at a 45 degree angle, and the investigator is faced with the problem of how to place stations such that corner and side lines precisely correspond to one another. The interstation distance either can be made the same for corner and side lines (as was the case in the present study), which results in corner line stations being away from the midlines of distance class bands, or different interstation distances must be used for corner vs side assessment lines. This problem is eliminated when a circular trap area is used, and the calculation of parameter estimates is made more straightforward.

An assumption common to all density estimation techniques is that of population closure, i.e. that the number of animals present within the study area remains constant during the period of data collection. Provided that the data are collected in a reasonably short period of time, the assumption of closure usually will not be seriously violated when mark-release trapping is used. Meeting the assumption of closure almost always is a problem when removal trapping is used. If ingress of animals occurs during or after the trapping period, the proportion of the population removed always will be under-estimated by some unknown amount and density will be over-estimated. Density independence is another important assumption when indices of activity and/or removal trapping are used to estimate density. If individual animal activity increases as density decreases, then density will be over-estimated, or, if animal activity decreases, then density will be under-estimated.

Wilson and Anderson (1985) used various models of home range utilization (bivariate normal, bivariate uniform, bivariate U-shaped, and truncated bivariate normal with random excursions) and dispersion patterns (random vs slightly clumped) in simulation studies of the trapping web density estimation technique, and found that these can affect the density estimate to varying degrees. For the method of analysis presented here, it is not currently known how different home range shapes, large differences among home range sizes, or different home range dispersion and/or utilization patterns affect the shape of the curve and the density estimate.

The method used to analyze assessment line data in the present study shows considerable promise for future studies. The method is more objective and more realistic than those used in previous studies, because the subjective delineation of zones, each with different but constant probabilities of capture, is eliminated. The problem of obtaining confidence intervals for the density estimate also has been eliminated, and population size and density always can be estimated for the same amount of area for any number of grids of a given size. The basic logic underlying the use of assessment lines for estimating density is sound; what is of particular interest, and remains to be seen, is how well the assessment line method performs relative to other estimators in cases where population densities are known.

Acknowledgments: We thank M. H. Smith for discussions on density estimation, and P. M. Dixon, J. M. Novak, and M. L. Taper for discussions on non-linear regression. J. H. Brown, N. J. Scott, Jr, 
M. H. Smith, and three anonymous referees read the manuscript and provided helpful comments and suggestions. During portions of the study, field assistance was provided by S. M. Bandoni and J. M. King. This study was supported by the Savannah River Ecology Laboratory Graduate Education Program and contract DE-AC09-76SROO819 between the United States Department of Energy and the University of Georgia.

\section{References}

Anderson D. R., Burnham K. P., White G. C. and Otis D. L. 1983. Density estimation of small-mammal populations using a trapping web and distance sampling methods. Ecology 64: 674-680.

Caswell H. 1989. Matrix population models: construction, analysis, and interpretation. Sinauer Associates Inc., Sunderland, Massachusetts: $1-328$.

Gorman M. L. and Stone R. D. 1990. The natural history of moles. Cornell Univ. Press, Ithaca, New York: $1-138$.

Harvey M. J. 1976. Home range, movements and diel activity of the eastern mole, Scalopus aquaticus. Am. Midl. Nat. 95: 436 - 445.

Moore A. W. 1940. A live mole trap. J. Mammal. 21: 223 - 225.

O'Farrell M. J., Kaufman D. W. and Lundahl D. W. 1977. Use of live-trapping with the assessment line method for density estimation. J. Mammal. 58: 575 - 582 .

Otis D. L., Burnham K. P., White G. C. and Anderson D. R. 1978. Statistical inference from capture data on closed animal populations. Wildl. Monographs 62: 1-135.

SAS Institute. 1985. SAS User's Guide: Statistics, Version 5 Ed. SAS Institute, Inc., Cary, North Carolina: 1 - 959 .

Smith M. H., Blessing R., Chelton J. G., Gentry J. B., Golley F. B. and McGinnis J. T. 1971. Determining density for small mammal populations using a grid and assessment lines. Acta theriol. 16: $105-125$.

Smith M. H., Gardner R. D., Gentry J. B., Kaufman D. W. and O'Farrell M. J. 1975. Density estimations of small mammal populations. [In: Small mammals: their productivity and population dynamics. F. B. Golley, K. Petrusewicz, and L. Ryszkowski, eds]. Cambridge Univ. Press, Cambridge: $25-53$.

Swift D. M. and Steinhorst R. K. 1976. A technique for estimating small mammal population densities using a grid and assessment lines. Acta theriol. 21: $471-480$.

Wheeler G. G. and Calhoun J. B. 1968. Manual for conducting ICSM census category 04 (octagon census and assessment traplines). International Census of Small Mammals Manual, No. 4, Parts 1 and 2, Edition 1: $1-50$.

White G. C., Anderson D. R., Burnham K. P. and Otis D. L. 1982. Capture-recapture and removal methods for sampling closed populations. LA-8787-NERP, UC-11. Los Alamos National Laboratory, Los Alamos, New Mexico: 1 - 235.

Wilson K. R. and Anderson D. R. 1985. Evaluation of a density estimator based on a trapping web and distance sampling theory. Ecology 66: 1185 - 1194 .

Yates T. L. and Schmidly D. J. 1977. Systematics of Scalopus aquaticus (Linnaeus) in Texas and adjacent states. Occas. Pap. Mus., Texas Tech Univ. 45: $1-36$.

Received 4 December 1992, accepted 14 July 1993 